\section{5 years of tracking physiotherapy evidence on PEDro, where are we now?}

\author{
Steven J Kamper, ${ }^{1}$ Anne M Moseley, ${ }^{1}$ Robert D Herbert, ${ }^{2}$ \\ Christopher G Maher, ${ }^{1}$ Mark R Elkins, ${ }^{3}$ Catherine Sherrington ${ }^{1}$
}

\section{BACKGROUND}

Healthcare systems across the globe have begun the transition to evidence-based practice. The degree to which the transition has occurred can be debated but, it is underpinned by a large and rapidly growing volume of research. However, this research is only useful if it is reliable, comprehensive and accessible.

The Physiotherapy Evidence Database (PEDro; http://www.pedro.org.au) recently celebrated 15 years of existence. PEDro is a free resource that indexes published randomised controlled trials (RCT), systematic reviews and clinical practice guidelines relevant to physiotherapy. The first physiotherapy RCT was published in 1929 and the first physiotherapy systematic review in 1982. Since then there has been exponential growth in the number of published RCTs and systematic reviews relevant to physiotherapy (figure 1). This editorial reflects on what PEDro tells us about the evidence base for physiotherapy and sports physiotherapy in particular.

\section{GROWTH IN RCTS, REVIEWS AND GUIDELINES}

As of October 2014, PEDro indexes 23049 RCTs, 5039 systematic reviews and 512 clinical practice guidelines. The largest number of studies are in the musculoskeletal

${ }^{1}$ Musculoskeletal Division, The George Institute for Global Health, Sydney Medical School, University of Sydney, Sydney, New South Wales, Australia;

${ }^{2}$ Neuroscience Research Australia (NeuRA), Sydney, New South Wales, Australia; ${ }^{3}$ Department of Respiratory Medicine, Royal Prince Alfred Hospital, Sydney, New South Wales, Australia

Correspondence to: Dr Steven J Kamper, Musculoskeletal Division, The George Institute for Global Health, Sydney Medical School, University of Sydney, PO Box M201 Missenden Road, Camperdown, NSW 2050, Australia; skamper@george.org.au subdiscipline (7676), followed by cardiothoracics (5334) and gerontology (4105). This volume of studies and the ongoing increase in rate of publication points to a substantial commitment to physiotherapy research.

There are 1325 sports physiotherapy studies (1098 RCTs, 222 systematic reviews and 5 practice guidelines); this is the second lowest of the 10 subdisciplines. The low number of studies is surprising given the high profile of sports physiotherapy. The rate of publications in the sports physiotherapy field is lower than in other subdisciplines.

\section{ACCESS AND SEARCHES ON PEDRO}

PEDro searches reflect engagement with the evidence by clinicians and researchers. In October 2014, 193662 searches were conducted on PEDro, roughly one search every $14 \mathrm{~s}$. PEDro has users from 215 countries and territories across the globe and indexes RCTs published in 29 guidelines indexed on PEDro. languages. The webpages are translated into 10 languages.

PEDro has two interfaces for searching: Simple Search and Advanced Search. The Advanced Search interface allows the user to select predetermined search terms within specific fields (such as 'Body Part', 'Problem', and type of 'Therapy') and/or to enter free-text search terms within specific fields (such as 'Author/Association', 'Abstract and Title', 'Published since' year, and 'Subdiscipline'). Where the 'Subdiscipline' field is used in PEDro searches, about 1.6\% of searches specify the 'Sports' subdiscipline.

\section{IMPLICATIONS FOR PRACTICE}

As part of the 15th anniversary of PEDro, users were asked to nominate the most influential physiotherapy RCTs. The nominations were judged by an international panel of experts to create a list of the top 15 physiotherapy RCTs based on strength of design and influence on clinical practice. The list included RCTs published in top-ranking general medical journals. Three RCTs directly relevant to sports physiotherapy were among the top 15 RCTs: a trial of extracorporeal shock wave therapy for plantar fasciitis, ${ }^{1}$ a trial of exercise and manual therapy versus 'wait-and-see' and steroid injections for tennis elbow, ${ }^{2}$ and a trial of exercises to prevent lower limb injuries in youth

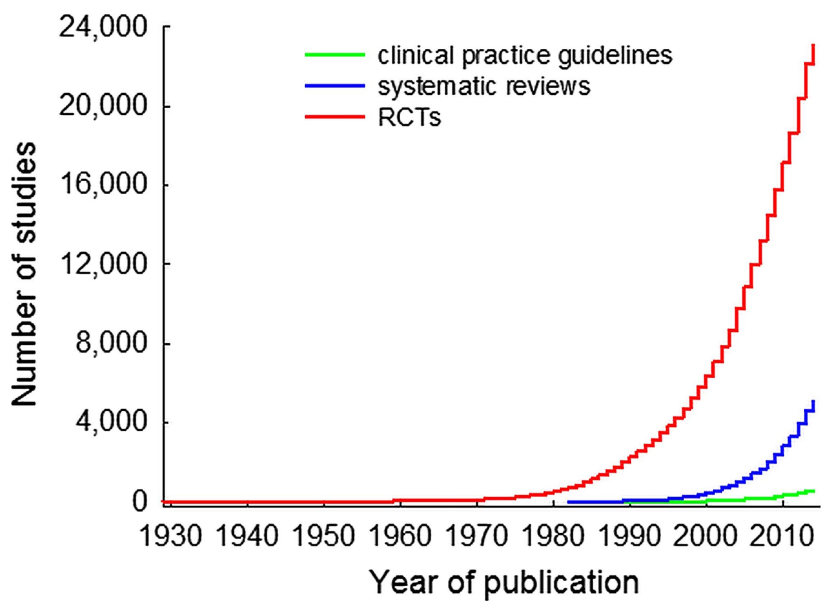

Figure 1 Growth in the number of published randomised controlled trials (RCTs), reviews and 
sports. $^{3}$ These three RCTs showcase two of the important functions of clinical research; the first demonstrated that a commonly used treatment for a prevalent problem was ineffective and the second and third evaluated interventions that are likely to have important beneficial clinical effects if implemented in the relevant population.

Consistent findings from wellconducted systematic reviews have clear implications for clinical practice. The best supported interventions in the physiotherapy field involve exercise. Strong evidence suggests that exercise interventions have important benefits for people with urinary incontinence, ${ }^{45}$ knee osteoarthritis, ${ }^{6}$ chronic obstructive pulmonary disease, ${ }^{7}$ older people at risk of falls, ${ }^{89}$ and preterm babies. ${ }^{10}$

\section{THE PEDRO SCALE}

A key feature of PEDro is the use of the PEDro scale to rate the methodological quality of RCTs. This scale helps readers quickly judge whether the trial results can be trusted and meaningfully interpreted. The PEDro scale includes 10 items that relate to internal validity and interpretability. The scale items are reported individually as well as summed to provide an overall indication of RCT quality. ${ }^{11}$ All RCTs are rated independently by two trained raters and any disagreements between the two are arbitrated by a third rater. The reliability and validity of the PEDro scale has been extensively demonstrated. ${ }^{12}$

The mean methodological quality score of all indexed RCT reports is 5.4 points on the 0-10 PEDro scale. ${ }^{13}$ PEDro scores have been improving by a mean of about 0.6 points per decade since $1960 .^{13}$ The individual PEDro scale items that are most commonly achieved are randomisation (96\%), between-group statistical comparisons (93\%) and mean and variability statistics for the group outcomes (89\%), while the least commonly achieved items were blinding of patients (7\%), blinding of therapists (2\%), intention-to-treat analysis (24\%) and concealed allocation (24\%). While blinding is difficult for many physiotherapy interventions, the other poorly-satisfied items could be targeted for improvement. In particular, intention-to-treat analysis and concealed allocation eliminate important sources of bias in the results of a RCT. ${ }^{14}{ }^{15}$ In sports physiotherapy, previous analyses ${ }^{16} \quad 17$ highlight areas of research design that could easily be improved: reporting of eligibility criteria and source of participants, reporting characteristics of participants at baseline, concealed allocation and intentionto-treat analysis.

\section{EXPECTATIONS AND HOPES FOR THE FUTURE}

The rapid growth in the volume and modest growth in quality of evidence, along with the frequency and geographical breadth of the use of PEDro reflect well on physiotherapy clinicians and researchers. However, there is room for improvement and development in a number of areas.

Some high profile subdisciplines, notably sports and paediatrics, are trailing others in terms of the number of studies being conducted and methodological quality. Efforts to increase research output relevant to clinical practice are encouraged. Ongoing work to improve the quality of research is important. To this end, the support of many journals for compulsory trial registration, ${ }^{18-20}$ increased publication of study protocols, ${ }^{21-24}$ and free access to research methods guidelines and reporting recommendations $^{25} 26$ are all positive developments.

A challenge for sports physiotherapy research relates to the extremely competitive funding environment across the world. Research in the sports field appears to attract less funding than other areas. This may have hampered study quality as the studies conducted are less likely to be well resourced and highly qualified and skilled researchers are not attracted and retained to sports injury research. Investment by funders in the key questions relevant to the field is needed to help address this challenge.

Improvements in training of researchers and an increase in the number of physiotherapy researchers gaining research degrees are also likely to increase the quality of published research over time. Allied to this is better integration of research and clinical practice to facilitate clinically relevant research and evidencebased practice. This can be assisted with online training tools, ${ }^{27} 28$ accessible texts and guides, ${ }^{29}$ courses at conferences and continuing professional development courses.

PEDro is undergoing ongoing development and enhancement with the aim of increasing access and uptake. Projects include a recent upgrade of the search function and development of training videos. There are plans to create direct links to trial registrations and trial protocols, make it possible to save searches, and to develop a more sophisticated search interface.
Contributors SJK wrote the first draft, all authors reviewed and provided input into successive drafts and approved the final version.

Competing interests The authors are directors of the Centre for Evidence-based Physiotherapy which is responsible for establishing and maintaining PEDro.

Provenance and peer review Not commissioned; internally peer reviewed.



To cite Kamper SJ, Moseley AM, Herbert RD, et al. Br J Sports Med 2015;49:907-909.

Accepted 16 March 2015

Published Online First 1 April 2015

Br J Sports Med 2015;49:907-909.

doi:10.1136/bjsports-2014-094468

\section{REFERENCES}

1 Buchbinder R, Ptasznik R, Gordon J, et al. Ultrasound-guided extracorporeal shock wave therapy for plantar fasciitis. A randomized controlled trial. JAMA 2002;288:1364-72.

2 Bisset L, Beller $E$, Jull G, et al. Mobilisation with movement and exercise, corticosteroid injection, or wait and see for tennis elbow: randomised trial. BMJ 2006;333:939.

3 Olsen OE, Myklebust G, Engebretsen L, et al. Exercises to prevent lower limb injuries in youth sports: cluster randomised controlled trial. BMJ 2005:330:449.

4 Dumoulin C, Hay-Smith EJ, Mac Habée-Séguin G. Pelvic floor muscle training versus no treatment, or inactive control treatments, for urinary incontinence in women. Cochrane Database Syst Rev 2014;5: CD005654.

5 Bø K, Herbert RD. There is not yet strong evidence that exercise regimens other than pelvic floor muscle training can reduce stress urinary incontinence in women: a systematic review. J Physiother 2013;59:159-68.

6 Fransen M, McConnell S. Exercise for osteoarthritis of the knee. Cochrane Database Syst Rev 2008;4: CD004376.

7 Lacasse Y, Goldstein R, Lasserson TJ, et al. Pulmonary rehabilitation for chronic obstructive pulmonary disease. Cochrane Database Syst Rev 2006;4:CD003793.

8 Gillespie LD, Robertson MC, Gillespie WJ, et al. Interventions for preventing falls in older people living in the community. Cochrane Database Syst Rev 2012;9:CD007146.

9 Sherrington C, Whitney J, Lord SR, et al. Effective exercise for the prevention of falls: a systematic review and meta-analysis. J Am Geriatr Soc 2008;56:2234-43.

10 Spittle A, Orton J, Anderson P, et al. Early developmental intervention programmes post-hospital discharge to prevent motor and cognitive impairments in preterm infants. Cochrane Database Syst Rev 2012;12:CD005495.

11 Maher CG, Sherrington C, Herbert RD, et al. Reliability of the PEDro scale for rating quality of randomized controlled trials. Phys Ther 2003;83:713-21.

12 Elkins MR, Moseley AM, Sherrington C, et al. Growth in the Physiotherapy Evidence Database (PEDro) and use of the PEDro scale. Br J Sports Med 2013;47:188-9.

13 Moseley A, Herbert RD, Maher CG, et al. Reported quality of randomized controlled trials of physiotherapy interventions has improved over time. J Clin Epidemiol 2011;64:594-601. 


\section{Editorial}

14 Elkins MR. Concealed allocation in randomised trials. J Physiother 2013;59:134-6.

15 Newell DJ. Intention-to-treat analysis: implications for quantitative and qualitative research. Int I Epidemiol 1992;21:837-41.

16 Kamper SJ, Moseley AM, Elkins MR. Clinical trials in sports physiotherapy. Building on five decades of research to produce even better trials: a critical review and tips for improvements. Br I Sports Med 2014;48:346-8.

17 Moseley AM, Elkins MR, Janer-Duncan L, et al. The quality of reports of randomized controlled trials varies between subdisciplines of physiotherapy. Physiother Can 2014;66:36-43.

18 Costa LO, Lin CW, Grossi DB, et al. Clinical trial registration in physiotherapy journals: recommendations from the International Society of Physiotherapy Journal Editors. J Physiother 2012;58:211-13.
19 Pinto RZ, Elkins MR, Moseley AM, et al. Many randomized trials of physical therapy interventions are not adequately registered: a survey of 200 published trials. Phys Ther 2012; 93:299-309.

20 De Angelis C, Drazen JM, Frizelle FA, et al Clinical trial registration: a statement from the International Committee of Medical Journal Editors. N Engl J Med 2004;351:1250-1.

21 Haines TP, Hush JM. Protocol papers published in Journal of Physiotherapy will strengthen the profession. J Physiother 2013;59:6.

22 Parreira PDCS, Costa LDCM, Takahashi R, et al. Do convolutions in Kinesio Taping matter? Comparison of two Kinesio Taping approaches in patients with chronic non-specific low back pain: protocol of a randomised trial. J Physiother 2013;59:52

23 http://www.biomedcentral.com/authors/protocols (accessed 17 Nov 2014).
24 Chien PF, Khan KS, Siassakos D. Registration of systematic reviews: PROSPERO. BJOG 2012;119:903-5.

25 Higgins JPT, Green S, eds. Cochrane handbook for systematic reviews of interventions version 5.0.2 [updated September 2009]. The Cochrane Collaboration, 2009. http://www. cochranehandbook.org

26 http://www.equator-network.org (accessed 17 Nov 2014).

27 https://www.youtube.com/playlist?list=PL5V_ VgcpCwuTL3RVpTIA1S5MzolnY7t0v (accessed 17 Nov 2014).

28 https://us.cochrane.org/understanding-evidencebased-healthcare-foundation-action (accessed 17 Nov 2014).

29 Herbert R, Jamtvedt G, Birger Hagen K, et al. Practical evidence-based physiotherapy. 2nd edn. Churchill-Livingstone Elsevier, 2011. 